\title{
Statistical Variations of Heart Orientation in Healthy Adults
}

\author{
Freddy Odille ${ }^{1,2}$, Shufang Liu ${ }^{1,3}$, Peter van Dam ${ }^{4}$, Jacques Felblinger ${ }^{1,2}$ \\ ${ }^{1}$ IADI, INSERM U947 and Université de Lorraine, Nancy, France \\ ${ }^{2}$ CIC-IT 1433, INSERM, CHRU Nancy and Université de Lorraine, Nancy, France \\ ${ }^{3}$ Technische Universität München, München, Germany \\ ${ }^{4}$ Radbound University Medical Center, Nijmegen, Netherlands
}

\begin{abstract}
The orientation of the heart in the chest impacts the shape and amplitude of surface ECG signals. It is also key information in electrocardiographic imaging (ECGI), where a model of the heart and torso is required. In this study we seek to analyze statistical relations between heart orientation and several easily available patient characteristics. Heart orientation data were obtained from an MRI database of 185 healthy adults. Relations with sex, height, weight (collected from case reports forms) and chest circumference (extracted from the images) were analyzed using univariate and multivariate linear regression. Chest circumference was found to be the best single predictor of heart orientation, and simple formulas were determined for its estimation. The proposed heart orientation statistical model might be used for selecting a torso/heart model from an existing database; this approach might allow ECGI techniques to be integrated into an ECG device.
\end{abstract}

\section{Introduction}

In electrocardiographic imaging (ECGI), a model of the heart and torso is required in order to reconstruct cardiac activation and recovery maps from surface ECG measurements [1], [2]. Such models can be obtained from imaging (e.g. MRI or CT); alternatively, when no imaging data from the patient is available, one may use a generic torso model or a model selected from an existing patient database [3]. The latter approach is of particular interest as it would allow ECGI techniques to be integrated into an ECG device. However criteria for selecting a plausible model need to be defined based on easily available patient characteristics. When selecting a torso model for ECGI, the position, size and orientation of the heart in the chest are key information. It has been shown that heart position and orientation can impact both the forward model and the inverse solution [4].

In a recent study [5], the heart size in 622 normal subjects was shown to be correlated with sex, age, height and weight. The authors proposed a multivariate linear model for estimating the heart size from these easily available patient data. Besides the size, the heart orientation remains an important degree of freedom in the torso model.

In this study we investigated the statistical relations between heart orientation and several easily available patient characteristics, including sex, age, weight, height and chest circumference. An MRI database of healthy adults was used for that purpose.

\section{Methods}

\subsection{Definition of heart orientation angles}

Heart orientation in the chest was described by two axes (see Fig. 1a): (i) the principal axis (or axis 1) was defined to be the longitudinal axis of the left ventricle, pointing towards the apex; (ii) the secondary axis (or axis 2) was defined to be, within a mid-cavity short-axis slice, the axis going from the center of the left ventricular (LV) cavity to the point of the right ventricular (RV) wall with maximal curvature (junction between the RV free wall and $\mathrm{RV}$ inferior wall).

The following angles were defined in order to describe the orientation of the two axes of the heart (see Fig 1b), using patient orientation terminology $(S=$ superior, $\mathrm{I}=$ inferior, $\mathrm{A}=$ anterior, $\mathrm{P}=$ posterior, $\mathrm{R}=$ right, $\mathrm{L}=\mathrm{left}$ ):

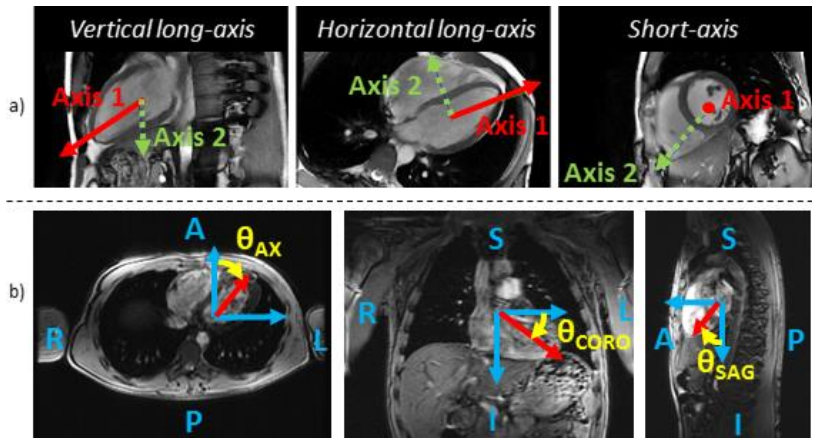

Figure 1 Definition of heart axes (a) and angles (b) 
- $\quad \theta_{A X}^{1}$ (respectively $\left.\theta_{A X}^{2}\right)$ : angle in degrees formed by the principal (respectively secondary) heart axis in the axial plane $\left(0^{\circ}=\mathrm{P}-\mathrm{A}\right.$ direction $)$

- $\theta_{S A G}^{1}$ (respectively $\theta_{S A G}^{2}$ ): angle in degrees formed by the principal (respectively secondary) heart axis in the sagittal plane $\left(0^{\circ}=\mathrm{S}-\mathrm{I}\right.$ direction)

- $\theta_{\text {CORO }}^{1}$ (respectively $\theta_{\text {CORO }}^{2}$ ): angle in degrees formed by the principal (respectively secondary) heart axis in the coronal plane $\left(0^{\circ}=\mathrm{R}-\mathrm{L}\right.$ direction)

\subsection{Cardiac MRI database}

Volunteers underwent an MRI scan on either a $1.5 \mathrm{~T}$ or a 3T Signa HDxt scanner (General Electric, Milwaukee, USA) at our institution between 2008 and 2016, as part of a general ethics protocol designed to test novel technical developments in MRI. This study was approved by an ethics committee and all subjects gave written informed consent. The volunteers were all adults with no selection criteria other than usual MRI contra-indications. All data were stored in our research database system ArchiMed [6].

Within this database, 185 subjects (122 males) who were imaged with cardiac scans in the 3 main anatomical planes (short axis, horizontal long axis and vertical long axis) were extracted.

The heart orientations were derived from the coordinates of the imaging planes prescribed by the radiographer (short-axis, horizontal and vertical long axes): the principal axis was calculated as the normal to the short-axis planes and the secondary axis was calculated as the intersection of short axis and horizontal long axis planes (see example in Fig. 1). The volunteers' sex, age, weight and height were collected from the case report forms.
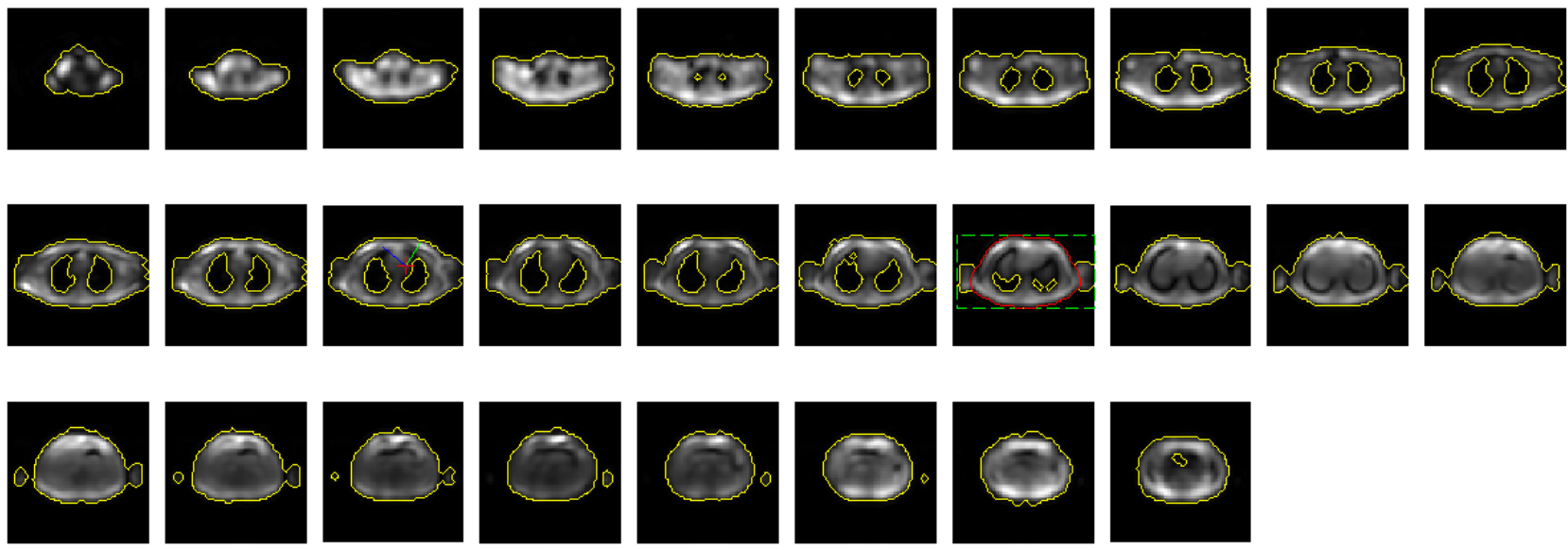

Figure 2 Exemplary low resolution images used for extracting chest circumference: body/lung masks were obtained by thresholding (yellow contours); then the lowest slice containing lung was selected (green rectangle); finally chest circumference was estimated from the body contour after a spline extrapolation in the arm regions (red contour). 


\section{Results}

\subsection{Heart orientation statistics}

Statistics of the population are summarized in Table 1. Standard deviations of the heart orientation angles, over the population, ranged between 9 and 32 degrees.

Table 1 Statistics of heart orientation in our volunteer database

\begin{tabular}{|c|c|c|}
\hline & Males & Females \\
\hline Number & 122 & 63 \\
\hline Age (years) & $32.6( \pm 11.5)$ & $29.6( \pm 10.8)$ \\
\hline Height $(\mathrm{cm})$ & $179.0( \pm 6.8)$ & $165.7( \pm 7.7)$ \\
\hline Weight (kg) & $74.2( \pm 9.1)$ & $61.2( \pm 11.5)$ \\
\hline Chest circumference $(\mathrm{cm})$ & $107.7( \pm 6.7)$ & $103.4( \pm 9.3)$ \\
\hline $\begin{array}{ll}\theta_{A X}^{1} & \text { (degrees) }\end{array}$ & $49.6( \pm 8.8)$ & $49.5( \pm 9.0)$ \\
\hline$\theta_{\text {CORO }}^{1}$ (degrees) & $35.7( \pm 10.3)$ & $36.9( \pm 10.1)$ \\
\hline $\boldsymbol{\theta}_{S A G}^{1}$ (degrees) & $49.7( \pm 12.2)$ & $48.8( \pm 10.6)$ \\
\hline $\begin{array}{ll}\theta_{A X}^{2} & \text { (degrees) }\end{array}$ & $51.6( \pm 22.0)$ & $49.6( \pm 17.2)$ \\
\hline$\theta_{\text {CORO }}^{2}$ (degrees) & $149.6( \pm 32.2)$ & $151.6( \pm 27.2)$ \\
\hline$\theta_{S A G}^{2}$ (degrees) & $79.4( \pm 30.1)$ & $77.0( \pm 29.9)$ \\
\hline
\end{tabular}

\subsection{Univariate linear regression}

Example linear fits obtained for the regression of the principal heart orientation axis in the coronal plane $\left(\boldsymbol{\theta}_{\text {CORo }}^{\boldsymbol{1}}\right)$ are shown in Fig. 3.

Detailed results for all angles are gathered in Table 2. The univariate analysis showed that the best single predictor of heart longitudinal axis is chest circumference, both for males and females. The most significant correlations were found for $\boldsymbol{\theta}_{S A G}^{1}$ and $\boldsymbol{\theta}_{\text {CORO }}^{1}$ angles ( $\mathrm{p}<0.003$ ), with goodness-of-fit $\mathrm{R}^{2}=0.14$ to 0.44 .

\subsection{Multivariate linear regression}

In the multivariate analysis of the form $\theta_{A X / S A G / C O R O}^{1 / 2}=a 0+a 1$. Age + a2. Height + a3. Weight + a4. ChestCircumference, the best fit of the data was found for $\boldsymbol{\theta}_{\boldsymbol{S} A G}^{\mathbf{1}}$, with $\mathrm{R}^{2}=0.43$ in males and $\mathrm{R}^{2}=0.21$ in females. However chest circumference was not found to be an independent predictor ( $p>0.1$ in all cases).

\section{Discussion}

For males, the univariate analysis shows that the best single predictor of heart principal axis orientation is the chest circumference. Correlations were significant for axial, coronal and sagittal angles. The best predictions are found for the sagittal and coronal angles, while the axial angles seem less predictable. For the secondary axis, correlations were not statistically significant. The circumference was not found to be an independent predictor in the multivariate analysis, meaning that chest circumference shares some information for predicting

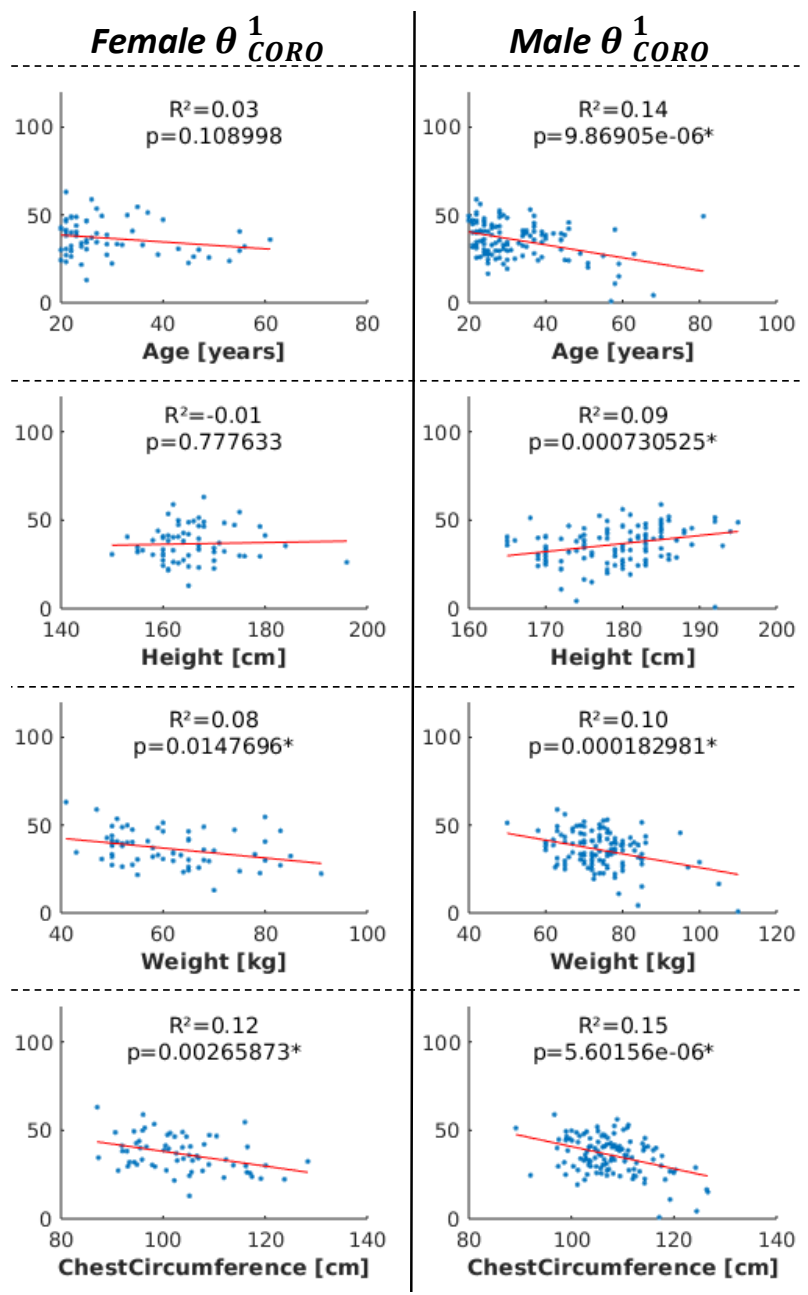

Figure 3 Univariate linear model fits for estimating the $\theta_{\text {CORO }}^{1}$ from the patient data (age, height, weight, chest circumference), for females (left) and males (right). Coefficients of determination $\mathrm{R}^{2}$ and $\mathrm{p}$ values are given, and * indicate a statistically significant relation.

orientation with age, height and weight.

For females, chest circumference is also the best single predictor, but it was significant for both principal and secondary axes of the heart. Correlations were significant for angles in coronal and sagittal planes only. Again, in the multivariate analysis, it was not found to be an independent predictor.

This study has limitations that should be mentioned. Our volunteer population contained more males than females, and the subjects were relatively young, with a high proportion of university students. We did not check that these subjects actually had no cardiopathy, but we assumed the majority of them was healthy.

The accuracy of the heart orientation angles was limited by the reproducibility of the manual placement of the imaging planes by the radiographers. While the longitudinal axis is fairly easy to define on the anatomy, the secondary axis is not always placed in the same way by different radiographers although they are instructed to 
Table 2 Univariate regression models for heart orientation estimation: $\mathbf{Y}=\mathbf{a 0}+\mathbf{a 1} . \mathbf{X}$

\begin{tabular}{|c|c|c|c|c|c|c|c|}
\hline \multirow{4}{*}{$\begin{array}{c}\mathrm{Y} \\
\boldsymbol{\theta}_{A X}^{1} \\
\text { [degrees] }\end{array}$} & \multirow[b]{2}{*}{$x$} & \multicolumn{3}{|c|}{ Males } & \multicolumn{3}{|c|}{ Females } \\
\hline & & a0 & a1 & p value & $\mathrm{a0}$ & a1 & p value \\
\hline & Age[years] & 53.926 & -0.134 & 0.064 & 44.901 & 0.162 & 0.15 \\
\hline & Height $[\mathrm{cm}]$ & 61.209 & -0.065 & 0.60 & 80.814 & -0.188 & 0.23 \\
\hline & Weight[kg] & 61.302 & -0.159 & 0.083 & 60.982 & -0.187 & 0.069 \\
\hline & ChestCircumference $[\mathrm{cm}]$ & 78.827 & -0.272 & $0.027^{*}$ & 60.016 & -0.101 & 0.44 \\
\hline \multirow{4}{*}{$\begin{array}{c}\theta_{\text {CORO }}^{1} \\
\text { [degrees] }\end{array}$} & Age[years] & 47.635 & -0.367 & $9.9 \mathrm{E}-06^{*}$ & 42.501 & -0.197 & 0.11 \\
\hline & Height $[\mathrm{cm}]$ & -45.347 & 0.456 & $0.00073 *$ & 28.413 & 0.050 & 0.78 \\
\hline & Weight $[\mathrm{kg}]$ & 64.861 & -0.390 & $0.00018^{*}$ & 53.847 & -0.282 & $0.015^{*}$ \\
\hline & ChestCircumference $[\mathrm{cm}]$ & 103.675 & -0.628 & $5.6 \mathrm{E}-06^{*}$ & 79.903 & -0.418 & $0.0027^{*}$ \\
\hline \multirow{4}{*}{$\begin{array}{c}\theta_{S A G}^{1} \\
\text { [degrees] }\end{array}$} & Age[years] & 36.111 & 0.420 & $6.9 \mathrm{E}-06^{*}$ & 45.311 & 0.117 & 0.37 \\
\hline & Height $[\mathrm{cm}]$ & 114.337 & -0.364 & $0.016^{*}$ & 20.907 & 0.168 & 0.36 \\
\hline & Weight $[\mathrm{kg}]$ & 8.940 & 0.550 & $2.2 \mathrm{E}-06^{*}$ & 19.022 & 0.482 & $2.7 \mathrm{E}-05^{*}$ \\
\hline & ChestCircumference $[\mathrm{cm}]$ & -49.987 & 0.925 & 1.0E-09* & -6.163 & 0.529 & $0.00025^{*}$ \\
\hline \multirow{4}{*}{$\begin{array}{c}\theta_{A X}^{2} \\
\text { [degrees] }\end{array}$} & Age[years] & 44.822 & 0.124 & 0.059 & 56.637 & -0.232 & 0.15 \\
\hline & Height $[\mathrm{cm}]$ & 23.424 & 0.143 & 0.048 & 26.727 & 0.139 & 0.54 \\
\hline & Weight $[\mathrm{kg}]$ & 42.235 & 0.090 & 0.047 & 54.158 & -0.073 & 0.63 \\
\hline & ChestCircumference $[\mathrm{cm}]$ & 36.901 & 0.111 & 0.047 & 68.884 & -0.184 & 0.32 \\
\hline \multirow{4}{*}{$\begin{array}{c}\theta_{\text {CORO }}^{2} \\
\text { [degrees] }\end{array}$} & Age[years] & 163.063 & -0.082 & 0.45 & 161.785 & -0.090 & 0.59 \\
\hline & Height $[\mathrm{cm}]$ & 110.604 & 0.278 & 0.45 & 112.882 & 0.279 & 0.25 \\
\hline & Weight $[\mathrm{kg}]$ & 156.982 & 0.045 & 0.44 & 140.718 & 0.305 & 0.051 \\
\hline & ChestCircumference $[\mathrm{cm}]$ & 178.338 & -0.167 & 0.45 & 115.561 & 0.420 & $0.034^{*}$ \\
\hline \multirow{4}{*}{$\begin{array}{c}\theta_{S A G}^{2} \\
\text { [degrees] }\end{array}$} & Age[years] & 80.313 & -0.276 & 0.15 & 72.520 & -0.091 & 0.75 \\
\hline & Height $[\mathrm{cm}]$ & 23.259 & 0.264 & 0.17 & 50.370 & 0.113 & 0.77 \\
\hline & Weight $[\mathrm{kg}]$ & 80.787 & -0.137 & 0.15 & 37.068 & 0.529 & $0.034 *$ \\
\hline & ChestCircumference $[\mathrm{cm}]$ & 106.669 & -0.330 & 0.14 & 0.822 & 0.658 & $0.029 *$ \\
\hline
\end{tabular}

* indicate statistically significant linear relation

follow the same guidelines in our institution. This may explain the larger variability of the secondary axis orientation angles and the less significant results compared to the principal axis. Respiratory motion is another source of variability for all these angles and was not taken into account here.

One possible use of the results presented in this study would be to create a model database for ECGI containing a reduced number of elements, yet covering the same range of angles as in the present population. Then for a given new subject/patient, one may use one of the proposed statistical models in Table 2 (our results suggest using chest circumference) to predict the heart orientation angles, and select the closest model from the database. If this model does not fit the data, then one may chose the second closest model, and so on.

\section{Conclusion}

Chest circumference is the best predictor of heart orientation. It is an easy-to-use index which embodies information from height and weight. It might be used to select a heart/torso model from a database for ECGI.

\section{Acknowledgements}

This study was funded by the European Commission through the Eurostars programme, grant number 9799 (ALVALE project: Anatomical Localization of the origin of Ventricular Arrhythmias from the 12-Lead ECG).

\section{References}

[1] C. Ramanathan, R. N. Ghanem, P. Jia, K. Ryu, et Y. Rudy, « Noninvasive electrocardiographic imaging for cardiac electrophysiology and arrhythmia », Nat. Med., vol. 10, $\mathrm{n}^{\circ}$ 4, p. 422-428, avril 2004.

[2] P. M. van Dam, T. F. Oostendorp, A. C. Linnenbank, et A. van Oosterom, «Non-invasive imaging of cardiac activation and recovery », Ann. Biomed. Eng., vol. 37, n 9, p. 17391756, sept. 2009.

[3] P. M. van Dam, R. Tung, K. Shivkumar, et M. Laks, "Quantitative localization of premature ventricular contractions using myocardial activation ECGI from the standard 12-lead electrocardiogram », J. Electrocardiol., vol. 46, n ${ }^{\circ}$ 6, p. 574-579, nov. 2013.

[4] G. J. M. Huiskamp et A. van Oosterom, « Heart position and orientation in forward and inverse electrocardiography », Med. Biol. Eng. Comput., vol. 30, nº 6, p. 613-620, nov. 1992.

[5] S. Pfaffenberger et al., « Size matters! Impact of age, sex, height, and weight on the normal heart size ", Circ. Cardiovasc. Imaging, vol. 6, nº 6, p. 1073-1079, nov. 2013.

[6] E. Micard, D. Husson, C.-I. Team, et J. Felblinger, " ArchiMed: A Data Management System for Clinical Research in Imaging », Front. ICT, vol. 3, 2016.

Address for correspondence:

Freddy Odille

IADI (Inserm U947) - CIC-IT 1433

Bâtiment Recherche, CHRU de Nancy Brabois, Rue du Morvan 54511 Vandoeuvre-lès-Nancy (France)

E-mail address: freddy.odille@inserm.fr 